\title{
Fostering Diversity in Top-Rated Pure Mathematics Graduate Programs
}

\section{Jana L. Gevertz and Joanna R. Wares}

The lack of a diverse student body continues to be a problem among graduate programs in pure mathematics. This is true despite broad agreement among scholars that a more diverse student body would greatly improve the graduate experience, as well as overall professional productivity [1]. In an article entitled "Prioritizing Diversity in Graduate School," Dr. Mark J. T. Smith, dean of the Graduate School and senior vice provost for academic affairs at The University of Texas at Austin, noted, "Diversity is a strength we should fervently embrace, as it allows us to leverage the rich perspectives, experiences and talents of the full spectrum of groups in our society" [1].

In this article, we explore what some top-rated pure mathematics graduate programs are doing to make their programs more diverse and inclusive. Most of the information in this article was gleaned from discussions with representatives from Columbia University, Harvard University, Massachusetts Institute of Technology, Princeton University, Stanford University, University of California Berkeley, and University of Chicago. Our aims are to report what these programs are doing, to give context to why these efforts are important, and to stimulate further discussion about diversity and inclusion in pure mathematics. This article is not directly applicable to related fields like applied mathematics and statistics (although trends in those fields are similar).

Jana L. Gevertz is a professor in the Department of Mathematics \& Statistics at The College of New Jersey. Her email address is gevertz@tcnj . edu.

Joanna R. Wares is an associate professor of mathematics at the University of Richmond. Her email address is jwares@richmond.edu.

For permission to reprint this article, please contact: reprint-permission aams.org.

DOI: https://dx.doi.org/10.1090/noti2026

\section{The Present State of Affairs}

A number of intractable aspects of the United States' educational system contribute to the continued underrepresentation of particular groups in graduate mathematics programs. These groups include, but are not limited to, women, people of color, sexual minorities, and gender minorities. Discussing the issue of increasing the number of women and, in particular, women of color in STEM, Dr. Dandrielle Lewis, associate professor of mathematics and director of liberal studies at the University of Wisconsin-Eau Claire states, "Contributing factors to the low representation include lack of positive and engaging STEM experiences, negative classroom experiences, lack of self-confidence in their mathematics skills, and 'chilly' campus and office climates" [2]. These factors result in low graduate mathematics application rates from historically underrepresented groups (HUGs).

Reports from the interviewed universities generally indicate a shortage of applicants from HUGs. Columbia University found that in its 2017-2018 graduate mathematics application pool, only 12 percent of applicants were women. Berkeley reported that, since 2016, approximately 18 percent of the graduate mathematics program applicants have been women. Chicago estimated that around 15 percent of its graduate mathematics applicants are women. Regarding underrepresented people of color, the institutions surveyed reported that rates of application and matriculation are significantly lower than their representation in the US population, but did not report exact numbers.

Low application rates yield low rates of admission and matriculation which, in turn, leads to individuals from HUGs earning mathematics doctorates at rates far below their demographic representation in society. With regard to gender diversity, the American Mathematical Society's 
(AMS's) Report on the 2016-2017 New Doctoral Recipients reveals that women who are US citizens were awarded 24.4 percent of pure math doctorates [3, Table D.4, II and III]. Moreover, US women fared worse at large private institutions, earning only 20 percent of mathematics $\mathrm{PhDs}$ awarded to US citizens [3, Table D.4, VII].

Correspondingly, the AMS report found that from 20162017, African American students received only 2.1 percent of the pure mathematics PhDs earned by US citizens ( 14 of 664) [3, Table D.4, II and III]. Large private institutions reported granting no pure mathematics doctorates to African American students [3, Table D.4, VII]. Hispanic/Latinx US citizens garnered less than 3 percent of pure mathematics PhDs (19 of 664) [3, Table D.4, II and III], with only two such degrees being granted from large private institutions [3, Table D.4, VII]. Hawaiian or Pacific Islanders earned less than 1 percent of such degrees ( 3 of 664) [3, Table D.4, II and III], and students of American Indian or Native Alaskan descent also earned less than 1 percent ( 2 of 664 ) [3, Table D.4, II and III]. Large private institutions did not award any PhDs to students of these backgrounds [3, Table D.4, VII].

Low rates of earned PhDs have a feedback effect. A lack of doctorates among HUGs leads to low rates of faculty hiring from HUGs, resulting in mathematics faculties that are fairly homogeneous. Dr. Helen Grundman, AMS's former director of education and diversity, has noted that having fewer relatable faculty yields fewer mentors, a probable factor in the depletion of diversity as you move up the ranks [4].

\section{Mechanisms for Change}

Initiatives for fostering diversity and inclusivity abound in academia, including at the top-rated mathematics graduate programs that we engaged. These initiatives can be broken down into three stages: the pre-application period, the admission process, and the post-acceptance departmental culture.

\section{The Pre-Application Period}

Effecting change before graduate school application time can be a powerful tool for recruiting a diverse class. Organizations like the National Association of Mathematicians (NAM) are focused on promoting the mathematical development of underrepresented minorities, and are a valuable resource for those looking to diversify their applicant pool. Dr. Edray Goins, professor of mathematics at Pomona College and president of NAM, is a fervent advocate of early outreach and recruitment. In his view, building relationships with historically black colleges and universities (HBCUs) is an important step in recruitment that many universities should utilize to increase diversity. Dr. Goins notes that although only around 10 percent of African American students attend HBCUs, nearly 40 percent of mathematics degrees granted to African American undergraduates come from those institutions.

In our discussions with Dr. Goins, he highlighted two ongoing programs focused on strengthening relationships between the community of mathematical scholars and historically underrepresented students: NAM's Undergraduate MATHFest [5] and the Field of Dreams Conference [6]. Funded by NAM, the Undergraduate MATHFest is an annual fall meeting held around the country on a rotating basis [5]. Although open to all students with aspirations of attending graduate school in the mathematical sciences, the focus of the symposium is on undergraduates from HBCUs. The meeting serves dual purposes: it provides attending students with a platform to present the results of their undergraduate research, and it provides attending universities a forum for meeting students and promoting their graduate programs. Dr. Goins believes that attending NAM's Undergraduate MATHFest is an excellent way for a university to send a strong message to African American students that they are welcome and wanted in their program.

Dr. Goins also highlighted the annual Field of Dreams Conference, sponsored by the Math Alliance [6]. The Math Alliance has a broad list of goals aimed at improving the diversity of doctoral degree holders in the mathematical sciences, and this conference represents just one of their initiatives. The Field of Dreams Conference focuses on first-generation students and those traditionally underrepresented in mathematics. Participants are informed about graduate programs in mathematics, meet with faculty members, and are advised on the application process, preparation for graduate level studies, and career opportunities. Graduate programs can recruit at the conference and invite student attendees to apply to their institutions. In addition, graduate programs that attend are given access to the names, contact information, CVs, and transcripts of all student participants. Actively inviting historically underrepresented students to apply sends a strong message of welcome to such students, and would likely help increase the diversity and strength of the applicant pool.

In addition to these conferences, the National Science Foundation funds a large number of REU programs to support undergraduate research at various host institutions. Dr. Goins views REUs that bring students from HUGs to campus as another useful recruitment tool, one that allows students to become familiar with particular institutions and programs before considering applying for advanced degrees.

We found that some of the surveyed institutions are using pre-application recruitment tools to increase the diversity of their applicant pool. For example, the University of Chicago as a whole recently instituted the Discover UChicago program. The program offers talented US students from historically underrepresented groups an expense-paid weekend at the university. There they can 
attend graduate admissions workshops, speak with the faculty and graduate students, and explore the graduate school itself [7]. The mathematics program successfully recruited one student who attended Discover UChicago to their 2019-2020 entering class. Chicago's mathematics department also uses an REU to recruit students from HUGs. Additionally, graduate students at the university have organized GRIT (Graduate Recruitment Initiative Team) to encourage undergraduates from HUGs at other universities to apply for graduate school at Chicago [8]. The GRIT program originated in biology, and mathematics graduate students are beginning to participate.

Similarly, MIT seeks to recruit a diverse student body through on-campus research opportunities. The universitywide MIT Summer Research Program [9] endeavors to increase the number of underrepresented minorities and underserved (e.g., low socio-economic background, first generation) students in research enterprises. Their Summer Program in Undergraduate Research (SPUR) in mathematics pairs one or two selected students with a graduate student mentor to work on a research project over a six-week period. To increase the diversity of students participating in that program, in 2017 they launched SPUR+ to specifically target students from HUGs who may not have the research background expected of SPUR participants [10]. SPUR+ begins three weeks prior to SPUR in order to provide guided reading in preparation for research.

To build a pipeline for admissions, MIT's and Stanford University's mathematics programs both report engaging in outreach efforts at early educational levels. For instance, Stanford University runs Math Circles at the elementary, middle, and high school levels, as well as a summer camp for high school students called SUMaC. Stanford reports that SUMaC has been an effective tool for recruiting outstanding talent, with a number of their top female mathematics majors having attended the camp. MIT has PRIMES (Program for Research in Mathematics, Engineering and Science) Circle, a free after-school math enrichment program for talented high school students living within commuting distance of Boston. The goal of this program is to increase diversity in the mathematical community by helping strong students from HUGs pursue their interest in mathematics and setting them on a path toward pursuing a math-based major in college. PRIMES Circle has about fifteen students per year, 80-90 percent of whom are women and 25 percent of whom are African American or Hispanic. MIT also has MathRoots, a two-week residential summer math camp for promising high school students from HUGs, selected from across the US. MathRoots' goal is to engage enthusiastic young mathematics students in creative problem solving by immersing them in a community of like-minded peers and mentors. The program has enrolled twenty students from HUGs annually over the past five years, with almost all of these students attending a four-year college and more than a quarter enrolling at MIT for their undergraduate studies.

Consistent with best practices encouraged by Dr. Goins, some of the top-rated mathematics programs actively recruit at local and national conferences that support undergraduate research and professional development for HUGs. Both MIT and Princeton report working with institutional partners to staff booths at the annual SACNAS (Society for Advancing Chicanos, Hispanics and Native Americans in Science) National Diversity in STEM Conference [11]. MIT uses this conference as an opportunity to invite students to apply to the MIT Summer Research Program.

\section{The Admissions Process}

With a more diverse applicant pool, the next step to consider when seeking to diversify a graduate cohort is how to evaluate applicants during the admissions process. Our conversations with university representatives revealed that the top-rated mathematics graduate programs are exploring how their admissions processes influence the diversity of their classes. Berkeley has altered the ranking of admission files by including broader impact scores in candidate rankings and de-emphasizing GREs. The university now uses a more holistic score: one that considers grades, letters of recommendation, and research experiences. It also educates its admission committee members about implicit bias before they review applications. The University of Chicago and Princeton University have also de-emphasized GRE scores and instead rely on holistic measures that account for student background when reviewing applications.

Of course, just because a program admits a diverse group of students does not mean that those students will choose to attend. The nature of the offer from the university plays a critical role in the student's decision-making process. Many of the interviewed programs emphasized the importance of making competitive offers to students from HUGs. For example, the math department at Berkeley is able to nominate fifteen students each year for a campus-wide internal competition for Berkeley and Chancellor Fellowships. These both come with two full years of fellowship support. Typically, 12-15 of the students nominated by the math department are offered the fellowship by the university, with 8-12 being offered to underrepresented students. Columbia University utilizes their Provost Diversity Fellowship, a one-time award of $\$ 8,000$ added to an offer, to attract students from HUGs.

\section{Departmental Culture}

In addition to the particulars of an offer, the culture of a university's mathematics graduate program and the success of its underrepresented students will bear on whether an offer of attendance will be accepted. Considerations of culture and success may well create a feedback mechanism in the acceptance of offers for graduate study. A program 
with a track record of progress in diversifying and graduating underrepresented students is likely to have an edge in appealing to students from HUGs. For example, Stanford's mathematics graduate student body is composed of 30 percent women, and they have attracted six of the last eight recipients of the Alice T. Schafer Mathematics Prize for Excellence in Mathematics by an Undergraduate Woman. Representatives from Stanford attribute these numbers to their progressive recruiting efforts.

As efforts to encourage HUGs to apply and matriculate start achieving positive results, mathematics graduate programs can also work to create an environment in which underrepresented students thrive. Representatives from many of the top-rated mathematics programs that we engaged mentioned that they are aware of, and actively addressing, issues of departmental culture. Some of the initiatives to help students from HUGs thrive begin as the students are preparing to embark on their graduate education. As examples, the University of California, Berkeley and Harvard University both have pre-first-year summer programs for HUGs. The program at Harvard provides admitted students coaching over the summer to prepare them for qualifying exams taken upon entering graduate school. Harvard reports that this program has worked well, with almost all of their students passing the qualifying exams at the beginning of their first year.

Dr. Ami Radunskaya, professor at Pomona College and past president of the Association for Women in Mathematics, has years of experience in the EDGE (Enhancing Diversity in Graduate Education) program. The EDGE program "seeks to create, identify and disseminate programs and strategies that improve the persistence of women and minority graduate students, and contribute to the development of a diverse mathematical community" [12]. From her discussions with students, she has learned the importance of fostering a collaborative culture among graduate students. Further, recognizing that students have a diversity of mathematical backgrounds, programs that offer opportunities to fill curricular gaps without stigma also foster the sort of supportive environment in which all students can thrive.

Several of the universities surveyed have programs at the departmental and institutional levels aimed at fostering an inclusive culture. At the department level, Columbia University has an active chapter of the Association of Women in Mathematics. MIT hosts a Women in Math Lunch Seminar series that brings together women graduate students, postdocs, faculty, and even some undergraduates to hear a senior female mathematician talk about her career and research. Berkeley and Stanford have active Noetherian Ring groups. Berkeley also described several other structural initiatives, including the formation of an Equity \& Inclusion Committee two years ago to evaluate its efforts to support a diverse student body. The mathematics department at
Berkeley has also created an in-house sexual harassment and violence prevention training workshop for new graduate students, run by graduate students. Stanford University has several departmental mentoring programs organized by graduate students and overseen by faculty. For instance, one of their programs pairs an incoming student with a more experienced graduate student for mentorship. Stanford's department also has the Stanford Women in Mathematics Mentoring program and a more general teaching-assistant mentoring program.

Several programs that support HUGs are institutional, rather than departmental-level, initiatives. Columbia University offers a Navigating Academia from the Margins workshop series through its Graduate School of Arts and Science's Office of Academic Diversity and Inclusion. Stanford University participates in the pilot Wellness Information Network for Graduate Students program, which includes wellness ambassadors who actively work with graduate students to ensure their general well-being. Princeton University's Graduate Scholars Program is designed to enhance and support the academic, social, and community development of HUGs during their first year of graduate studies. To ensure that the institution follows up with these students after their first year, the Access, Diversity, and Inclusion (ADI) team offers workshops on academic skills, time management, self-care, and financial planning, as well as informal social activities to support HUGs across academic disciplines. The deans of the ADI advise graduate students, supporting them through key milestones and guiding them toward completion and life beyond Princeton.

\section{In Summary}

Overall, our conversations with representatives from seven of the top-rated pure mathematics graduate departments revealed that the diversity of their graduate student body is important to each of them. We found that their initiatives operate on many levels: in how they shape the applicant pool through outreach initiatives, in developing more holistic admissions criteria, and in reshaping the culture of their mathematics department to be more inclusive of HUGs. With continued attention, we can work toward building a mathematics community that more accurately reflects the demographics of the nation.

The authors would like to thank Dr. Edray Goins and Dr. Ami Radunskaya, as well as the departmental representatives from each of the universities listed, for providing information for this article. 


\section{COMMUNICATION}

\section{References}

[1] Smith MJT. Prioritizing Diversity in Graduate Education, 2018. Retrieved from https://diverseeducation.com /article/129336.

[2] Lewis D. Increasing diversity and inclusion for women in STEM, Notices Amer. Math. Soc., 62(8), 2015.

[3] Annual Survey of the Mathematical Sciences. Demographics in the Mathematical Sciences: New PhDs, Gender \& Citizenship, https://www.ams.org/profession/data annual-survey/demographics

[4] Kennedy S. A Conversation with Helen Grundman, AMS Director of Education and Diversity, Notices Amer. Math. Soc., 63(11), 2016.

[5] National Association of Mathematicians. Undergraduate MATHFest, https://www. nam-math.org/mathfest.html.

[6] The National Alliance for Doctoral Studies in the Mathematical Sciences. Field of Dreams Conference, https:// matha11iance.org/field-of-dreams-conference.

[7] University of Chicago. Discover UChicago program, https://grad.uchicago. edu/admissions/diversity /discover.

[8] University of Chicago. Graduate Recruitment Initiative Team, https://biosciences.uchicago.edu/diversity Varit.

[9] Massachusetts Institute of Technologv. MIT Summer Research Program, https://oge.mit.edu/graddiversity /msrn.

[10] Massachusetts Institute of Technology. Summer Program in Undergraduate Research of the MIT Mathematics Department, https://math.mit.edu/research/undergraduate /spur/index.php

[11] Soclety for Advancement of Chicanos/Hispanics and Native Americans in Science. The National Diversity in STEM Conference, https://www. sacnas . ord/what-we-do /conference

[12] The Edge Program. https://www. edgeforwomen . org.

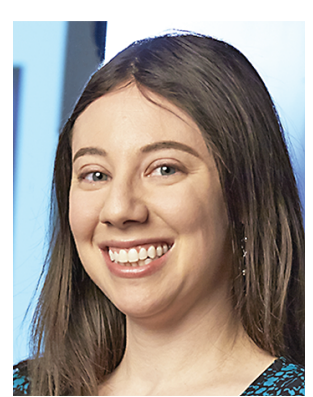

Jana L. Gevertz

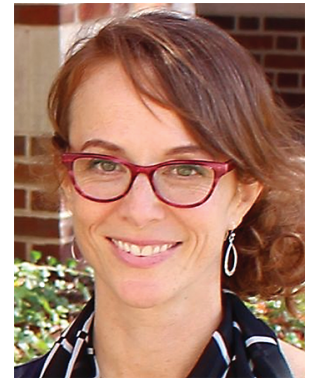

Joanna R. Wares

\section{Credits}

Photo of Jana L. Gevertz is courtesy of The College of New Jersey.

Photo of Joanna R. Wares is courtesy of University of Richmond.
FEATURED TITLE FROM

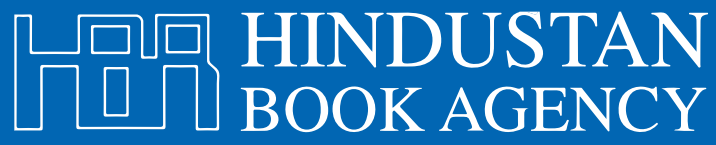

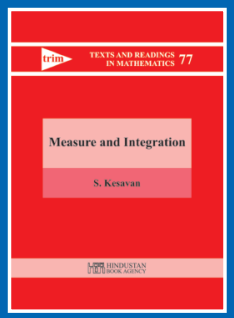

\section{Measure and} Integration

\section{S. Kesavan}

This book deals with topics usually studied in a master's or graduate level course on the theory of measure and integration. It starts with the Riemann integral and points out some of its shortcomings which motivate the theory of measure and the Lebesgue integral.

Starting with abstract measures and outer-measures, the Lebesgue measure is constructed and its important properties are highlighted. Measurable functions, different notions of convergence, the Lebesgue integral, the fundamental theorem of calculus, product spaces, and signed measures are studied. There is a separate chapter on the change of variable formula and one on $L p$-spaces.

Most of the material in this book can be covered in a one-semester course. The prerequisite for following this book is familiarity with basic real analysis and elementary topological notions, with special emphasis on the topology of the $N$-dimensional euclidean space. Each chapter is provided with a variety of exercises.

Hindustan Book Agency; 2019; 252 pages; Hardcover; ISBN: 978-93-86279-77-4; List US\$45; AMS members US\$36; Order code HIN/77; jookstore.ams.org/hin-77

Titles published by the Hindustan Book Agency (New Delhi, India) include studies in advanced mathematics, monographs, lecture notes, and/or conference proceedings on current topics of interest.

Discover more books at bookstore.ams.org/hin.

Publications of Hindustan Book Agency are distributed within the Americas by the American Mathematical Society. Maximum discount of $20 \%$ for commercial channels.

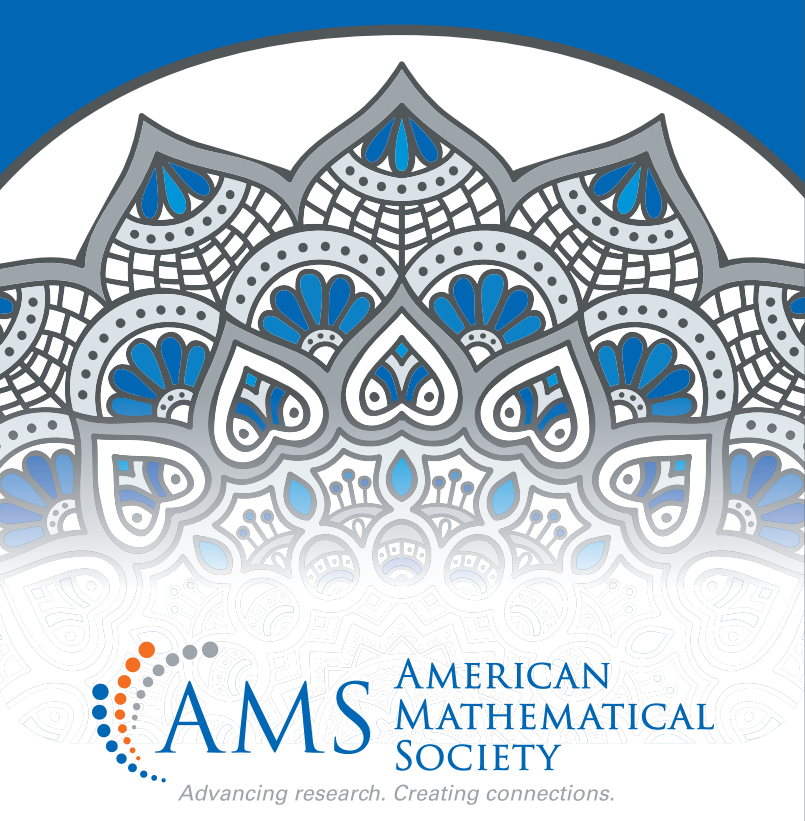

